\title{
Self-Consistent Embedding of Density-Matrix Renormalization Group Wavefunctions in a Density Functional Environment
}

\author{
Thomas Dresselhaus, ${ }^{1}$ Johannes Neugebauer, ${ }^{1, a)}$ Stefan Knecht, ${ }^{2, b}$ Sebastian Keller, ${ }^{2}$ Yingjin Ma, ${ }^{2}$ and Markus \\ Reiher $^{2, \mathrm{c})}$ \\ ${ }^{1)}$ Westfälische Wilhelms-Universität Münster, Theoretische Organische Chemie, \\ Organisch-Chemisches Institut and Center for Multiscale Theory and Computation, Corrensstraße 40, \\ 48149 Münster, Germany \\ ${ }^{2)}$ ETH Zürich, Laboratorium für Physikalische Chemie, Vladimir-Prelog-Weg 2, 8093 Zürich, \\ Switzerland
}

We present the first implementation of a density matrix renormalization group algorithm embedded in an environment described by density functional theory. The frozen density embedding scheme is used with a freeze-and-thaw strategy for a self-consistent polarization of the orbital-optimized wavefunction and the environmental densities with respect to each other.

Chemical reactions are local phenomena, in which usually only one or two bonds are formed or broken at a time. For this reason, the cluster approach was established in computational chemistry, in which the atomistic rearrangements under study are embedded in a structural model that is as large as necessary to comprise all electronic effects on the reactive subsystem (see, for instance, the extensive work on such cluster models for active sites of metalloproteins by Siegbahn, Himo, and co-workers ${ }^{1}$ ). In general, long-range electrostatic interactions may not be neglected and schemes have been devised to incorporate them (most prominent are quantummechanical/molecular-mechanical embedding ${ }^{2}$ and polarizable continuum models $\frac{3}{3}$ ). For the appropriate description of extensive electronic coupling of a subsystem to its environment (mediated, for example, through $\pi$ conjugated molecular substructures), more involved embedding schemes are needed.

Clearly, the embedding of a quantum system of interest into a more or less electronically noninnocent environment is the realm of open-system quantum theory $\underline{\underline{4}}$. The central quantity of this well-known theory is the reduced density matrix of a subsystem rather than the wave function of a stationary state. This formalism has recently been adapted for an embedding framework $\stackrel{5.6}{=}$ However, it is not straightforward to employ (for instance, the environment had to be approximated in a mean-field fashion). Practical suggestions for the applicability to large chemical systems have been made ${ }^{\underline{7}}$

Considering the success of density functional theory (DFT) for routine applications in computational chemistry, a feasible approach that is able to describe chemically complex and structured environments should also be based on DFT. In the frozen density embedding 8 (FDE) scheme, which is based on subsystem DFT,,$\frac{9,10}{, 9}$ a subsystem is calculated in a Kohn-Sham (KS) approach by adding an effective potential to the KS po-

\footnotetext{
a) Electronic mail: j.neugebauer@uni-muenster.de

b) Electronic mail: stefan.knecht@phys.chem.ethz.ch

c) Electronic mail: markus.reiher@phys.chem.ethz.ch
}

tential of the isolated subsystem. It takes into account all interactions with the environment, assuming a constant (frozen) environmental electron density. In FDE not only the exchange-correlation energy contribution, but also the non-interacting kinetic energy term is calculated from an approximate explicit density functional. No knowledge of orbitals of the supersystem is needed for an FDE/subsystem DFT calculation, nor do the orbitals of the active subsystem need to be orthogonal to those of the environmental subsystems. In this way large environments can be considered.

Due to the limited accuracy of kinetic energy functionals for chemical systems, FDE cannot be directly applied to subsystems which are connected via bonds with covalent character $\stackrel{11}{ }$. Extensions to resolve this limitation are available. Often, potential-reconstruction methods have been proposed in this context $\underline{12}-16$ A related embedding scheme by Manby at al $\stackrel{17}{\underline{1}}$ resolves this issue by imposing orthogonality constraints between orbitals of the active subsystem and the environment. This strategy avoids the need for explicit density functionals for the evaluation of the kinetic energy. KS orbitals, which can be conveniently obtained from comparatively cheap DFT calculations, are employed here. Furthermore, a straightforward basis-set truncation scheme, needed for a reduction of the computational cost for the subsequent wavefunction calculation, is readily available $\stackrel{18}{=} \mathrm{In} \mathrm{FDE}$, all electron-correlation effects between different subsystems are captured by the density functional. In principle, all correlation effects, including the polarization of the wavefunction of the active subsystem, can be accurately described for the (admittedly unknown) exact functional. Considering the fact that at times approximate density functionals can yield rather inaccurate results, it is desirable to describe an (active) subsystem by systematically improvable wavefunction theory (WFT). Although based on DFT, the FDE framework also allows for treating one subsystem with a correlated wavefunction method and the rest of the system with DFT $\underline{19}$. Several research groups implemented such a WFT-in-DFT embedding; for instance, to facilitate complete-active-space self-consistent field (CASSCF) in periodio ${ }^{20,21}$ and non- 
periodic 22 DFT, Møller-Plesset perturbation theory in $\mathrm{DFT}^{23}$, coupled cluster in DFT $\stackrel{24}{ }$, CASSCF with secondorder perturbation theory (CASPT2) in DFT 22,25 , and most recently quantum Monte Carlo in $\mathrm{DFT}^{26}$ calculations (for an overview see Refs. 27 29).

Within a comparatively short time, the density matrix renormalization group (DMRG) alorithm ${ }^{30,31}$ has become a standard WFT approach in quantum chemistry (for reviews see Refs. 32 37), which is under continuous development (see Refs. 38 39 for most recent work) providing interesting new perspectives for the description of electronic structures dominated by static electron correlation $\underline{40}-\underline{48}$ DMRG is able to iteratively converge to the exact solution of the electronic Schrödinger equation in a given active orbital space with polynomial rather than factorial cost by which the full configuration (and thus the CASSCF) approach is plagued. However, the benefits of this iterative protocol come with a caveat that has long prohibited the extension of its applicability to compact and strongly correlated molecules like transition metal complexes and clusters. This caveat is rooted in the matrix-product-state structure of the optimized DMRG wave function. As a consequence, DMRG should only be applied to pseudo-one-dimensional molecular structures. It could be shown $\underline{49}$ that its benefits outweigh these formal objections; still, care must be taken to control the parameters that determine the accuracy of an iterative DMRG calculation ${ }^{50}$. It has now been fully recognized that accuracy- and performancewise, DMRG can be a substitute for the CASSCF (and CASPT2) methodology pioneered by the Lund group 51 for cases, which require much larger active orbital spaces than those accessible by this standard approach. With DMRG, active orbital spaces are in reach that are about five times larger than those accessible to CASSCF. This feature makes DMRG an ideal target for a multireference WFT-in-DFT embedding method.

Here, we report the first implementation of a DMRGin-DFT embedding (including orbital relaxation in the DMRG step; DMRG-SCF), which follows the FDE framework in a freeze-and-thaw $\underline{52}$ (f\&t) manner, to establish a fully self-consistent embedding. Often (but not always ${ }^{26}$ ) is the environment polarized by employing a DFT-in-DFT approach, then kept fixed in the WFT calculations, and a fixed embedding potential is applied 22.24 . Alternatively, Carter and co-workers imposed constraints on the total ${ }^{53}$ or environmental ${ }^{21}$ density.

In our implementation an active subsystem (act) calculated with a WFT method is embedded into an environment (env) described with DFT and the following steps are carried out:

1) Perform DFT calculations on the isolated subsystems to calculate initial densities ${ }^{(0)} \rho^{\text {env }}$ and ${ }^{(0)} \rho^{\text {act }}$

2) Calculate an embedding potential for the environmental subsystem ${ }^{(n)} v_{e m b}^{\text {env }}\left[{ }^{(n-1)} \rho^{\text {env }},{ }^{(n-1)} \rho^{\text {act }}\right]$

3) Perform an embedded DFT calculation on the environmental subsystem: ${ }^{(n)} v_{e m b}^{\text {env }} \rightarrow{ }^{(n)} \rho^{\text {env }}$

4) Calculate an embedding potential for the active sub- system $\left.{ }^{(n)} v_{e m b}^{\text {act }}{ }^{[(n-1)} \rho^{\text {act }},{ }^{(n)} \rho^{\text {env }}\right]$

5) Perform an embedded WFT calculation on the active subsystem: ${ }^{(n)} v_{e m b}^{\text {act }} \rightarrow{ }^{(n)} \rho^{\text {act }}$

6) Repeat 2.-5. (increasing $n$ by 1 each time) until convergence to a given threshold is reached

WFT methods accessible in our implementation include Hartree-Fock, CASSCF, DMRG (with and without orbital optimization: DMRG-SCF and DMRG-CI, resp.). Although the embedding potential for the WFT subsystem depends on the density calculated from the wavefunction, we keep it fixed during the wavefunction calculation, which simplifies the implementation. The error introduced by this approximation vanishes as soon as the wavefunction converges w.r.t. the f\&t procedure, thus no additional error is produced in the final results.

The implementation provides an interface between a development version of MOLCAS ${ }^{54}$, the MAQUIS quantum chemical DMRG program, 55 and ADF $56-58$. The quantum chemical version of MAQUIS explicitly implements a matrix-product operator based formalism and exploits all technical features of the underlying MAQUIS ${ }^{59}$ program. Starting orbitals for the DMRG calculations were taken from a preceding Hartree-Fock calculation with MoLCAS. We extended the scripting framework PyADF 60 accordingly to allow for easy scripting of the calculations. All DFT calculations were performed with ADF and the PW91 $\underline{61}$ functional in a TZP $\underline{\underline{62}}$ basis. Also the embedding potentials were calculated with ADF with the PW91k ${ }^{63}$ functional for the nonadditive kinetic energy. A supermolecular integration grid was used throughout. All DMRG-SCF calculations were performed with MoLCAS using a cc-pVTZ ${ }^{64}$ basis. The number of renormalized DMRG block states $M$ was set to 1000 , unless otherwise noted. Structures were optimized with $\mathrm{ORCA}^{65}$ with BP86 66,67-D3BJ $\underline{68} /$ Def2-TZVP $\underline{\text { 69 }}$ and density fitting.

As a first technical test, for which we can still obtain CASSCF reference data, we considered the polarization of a methane molecule embedded in the environment of an ammonium ion as depicted in Figure 1. All DMRGSCF results for an active space of eight electrons (the $1 s$ electrons of $\mathrm{C}$ are kept frozen) in eight orbitals, $\mathrm{CAS}(8,8)$, are compiled in Table I. At equilibrium distance ('eq.'), the effect on the subsystem energy is by an order of magnitude more pronounced than at a distance of $10 \AA$ between the molecule centers. As expected, the polarization of the wavefunction is more pronounced for the calculation on a closer distance. The wavefunction-polarization effect is more obvious when investigating the dipole moment of the methane molecule as shown in Table ogous CASSCF-in-DFT calculations (not shown) yield the same results up to the presented accuracy. Furthermore, the DMRG-SCF-in-DFT data compares very well with the corresponding DFT-in-DFT results (right column of Table II).

To demonstrate that our new implementation reaches beyond the capabilities of a CASSCF-in-DFT embedding approach, we studied the dipole moment of a HCN molecule induced by the presence of a second HCN 
TABLE I. DMRG(8,8)-SCF-in-DFT calculations on methane embedded in an ammoniuim-ion environment at different distances. Energies are given in Hartree. $E^{\text {act }}$ is the energy of the WFT subsystem according to Huang and Carter ${ }^{14}$. In the last column, the energy change due to the polarization of the wavefunction is shown.

\begin{tabular}{lcc}
\hline \hline $\boldsymbol{r}(\mathrm{C}-\mathrm{N})$ & $E^{\text {act }}-E^{\text {iso }}$ & $\left(E^{\text {act }}-\int \rho^{\text {act }}(\boldsymbol{r}) v_{\mathrm{emb}}^{\text {act }}(\boldsymbol{r}) d \boldsymbol{r}\right)-E^{\text {iso }}$ \\
\hline $10 \AA$ & $-5.31 \times 10^{-1}$ & $7.44 \times 10^{-5}$ \\
5.68 (eq. $) \AA-9.34 \times 10^{-1}$ & $5.97 \times 10^{-4}$ \\
\hline \hline
\end{tabular}
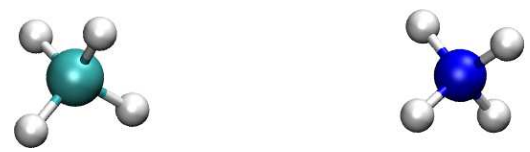

FIG. 1. A methane molecule (left) in the presence of an ammonium ion (right) at the equilibrium distance of $5.68 \AA$.

TABLE II. Comparison of dipole moments in Debye of methane embedded in an ammonium-ion environment obtained from DMRG(8,8)-SCF-in-DFT and analogous DFTin-DFT calculations, respectively.

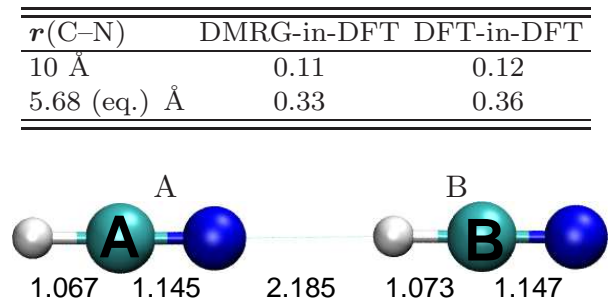

FIG. 2. The supermolecular HCN dimer consisting of two HCN molecules denoted A and B (distances are in $\AA$ ).

molecule (Figure 2). We selected this HCN dimer as an appropriate benchmark example as it is known that HCN chains show a strong cooperative behaviour in this regard $\stackrel{27}{i}$ Table III comprises the calculated dipole moments obtained using either a supermolecular or a WFT/DFT-in-DFT approach. The reference DMRGSCF calculation on the supermolecular dimer comprised an active space of 20 electrons in 18 orbitals (twice the valence complete active space for one $\mathrm{HCN}$ molecule, i.e., a CAS $(10,9))$. We observe only small differences in the dipole moments between the DFT and the WFT calculations. A qualitatively correct polarization of the embedded HCN molecule compared to its isolated counterpart can be observed. An advantage of the subsystem approach is the possibility to investigate the properties of only one subsystem in the presence of other subsystems. To demonstrate this feature, we collected the different dipole moments for the two different $\mathrm{HCN}$ molecules A and B in Table III The importance of taking into account a fully self-consistent polarization of the environment by means of a f\&t procedure is indicated in Figure 3. The f\&t procedure shows a fast linear convergence. The error decreases by remarkable $98.6 \%$ in each f\&t cycle compared to the converged result. Our new DMRGin-DFT embedding approach combines the advantages
TABLE III. The dipole moment of $\mathrm{HCN}$ in the presence or absence of another HCN molecule. Supermolecular results are compared to those from embedding calculations, in which the environment was polarized by a density taken either from a wavefunction or from a DFT calculation, respectively.

\begin{tabular}{lccc}
\hline \hline Method & $\begin{array}{c}\text { Active } \\
\text { fragment }\end{array}$ & $\begin{array}{c}\text { Polarization of } \\
\text { the environment }\end{array}$ & $\begin{array}{c}\text { Dipole moment } \\
\text { (active fragment) }\end{array}$ \\
\hline DFT & isolated & - & 2.95 \\
DMRG(10,9)-SCF & isolated & - & 3.08 \\
DFT & dimer & - & $3.38^{1}$ \\
DMRG 2 (20,18)-SCF & dimer & - & $3.42^{1}$ \\
DMRG(10,9)-SCF & $\mathrm{A}$ & DMRG & 3.42 \\
DMRG(10,9)-SCF & $\mathrm{A}$ & DFT & 3.39 \\
DFT & $\mathrm{A}$ & DFT & 3.39 \\
DMRG(10,9)-SCF & $\mathrm{B}$ & DMRG & 3.29 \\
DMRG(10,9)-SCF & $\mathrm{B}$ & DFT & 3.30 \\
DFT & $\mathrm{B}$ & DFT & 3.30 \\
\hline \hline
\end{tabular}

${ }^{1}$ Per molecule.

2 DMRG parameter $M$ was increased to 2048.

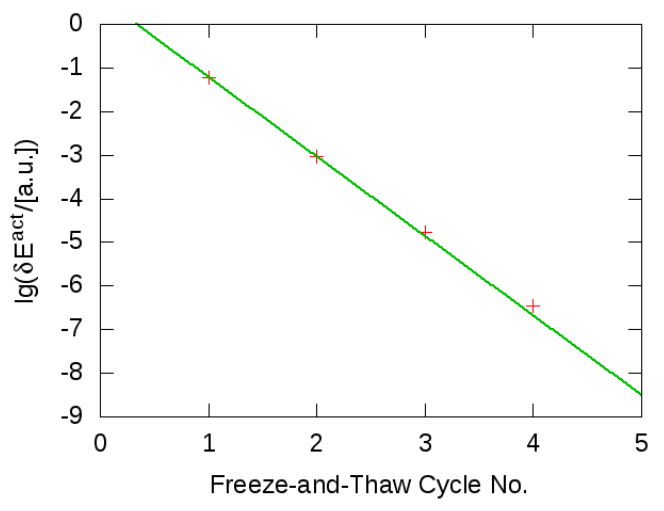

FIG. 3. Error of the total electronic energy in Hartree with respect to a converged f\&t procedure for a DMRG(10,9)-SCFin-DFT calculation on a HCN molecule embedded into the environment of another $\mathrm{HCN}$ molecule as a function of the f\&t cycle number.

of DMRG with an accurate quantum mechanical embedding scheme using a f\&t approach for the polarization of the embedding density. This approach facilitates accurate calculations on systems with strong static correlation embedded in environments whose effects are important beyond a classical (purely electrostatic) description. The pilot applications reported in this communication are proof-of-principle calculations and the algorithms offers various options for future developments. Clearly, perturbative corrections should be considered on top of the converged DMRG-in-DFT results to capture dynamic correlations. The MAQUIS program is currently extended to produce the required many-body density matrices for different perturbation theories to second order.

Acknowledgements TD and JN acknowledge funding by the Deutsche Forschungsgemeinschaft through SFB 858. This work was supported by ETH Zürich (Research Grant ETH-34 12-2) and by the Schweizerischer Nation- 
alfonds (project 200020_144458). Support by COST Action CODECS is gratefully acknowledged.

${ }^{1}$ P. E. Siegbahn and F. Himo, WIREs Comput. Mol. Sci. 1, 323 (2011).

${ }^{2}$ H. M. Senn and W. Thiel, Angew. Chem. Int. Ed. 48, 1198 (2009).

${ }^{3}$ B. Mennucci, WIREs Comput. Mol. Sci. 2, 386 (2012).

${ }^{4}$ A. Amann and U. Muller-Herold, Offene Quantensysteme: Die Primas Lectures, Springer-Verlag, Berlin, Heidelberg, 2011.

${ }^{5}$ G. Knizia and G. K.-L. Chan, Phys. Rev. Lett. 109, 186404 (2012).

${ }^{6}$ G. Knizia and G. K.-L. Chan, J. Chem. Theory Comput. 9, $1428(2013)$

${ }^{7}$ Q. Sun and G. K.-L. Chan, J. Chem. Theory Comput., DOI: 10.1021/ct500512f (2014).

${ }^{8}$ T. A. Wesolowski and A. Warshel, J. Phys. Chem. 97, 8050 (1993).

${ }^{9}$ G. Senatore and K. R. Subbaswamy, Phys. Rev. B 34, 5754 (1986).

${ }^{10}$ P. Cortona, Phys. Rev. B 44, 8454 (1991).

${ }^{11} \mathrm{~S}$. Fux, K. Kiewisch, C. R. Jacob, J. Neugebauer, and M. Reiner, Chem. Phys. Lett. 461, 353 (2008).

${ }^{12}$ O. Roncero, M. P. de Lara-Castells, P. Villarreal, F. Flores, J. Ortega, M. Paniagua, and A. Aguado, J. Chem. Phys. 129, 184104 (2008).

${ }^{13}$ J. D. Goodpaster, N. Ananth, F. R. Manby, and T. F. Miller, J. Chem. Phys. 133, 084103 (2010).

${ }^{14}$ C. Huang and E. A. Carter, J. Chem. Phys. 135, 194104 (2011).

${ }^{15}$ C. R. JACOB and L. Visscher, J. Chem. Phys. 128, 155102 (2008).

${ }^{16}$ S. Fux, C. R. Jacob, J. Neugebauer, L. Visscher, and M. ReiHER, J. Chem. Phys. 132, 164101 (2010).

${ }^{17}$ F. R. Manby, M. Stella, J. D. Goodpaster, and T. F. Miller, J. Chem. Theory Comput. 8, 2564 (2012).

${ }^{18}$ T. A. Barnes, J. D. Goodpaster, F. R. Manby, and T. F. Miller, J. Chem. Phys. 139, 024103 (2013).

${ }^{19}$ T. A. Wesolowski, Phys. Rev. A 77, 012504 (2008).

${ }^{20}$ T. Klüner, N. Govind, Y. A. Wang, and E. A. Carter, Phys, Rev. Lett. 86, 5954 (2001).

${ }^{21}$ P. Huang and E. A. CArter, J. Chem. Phys. 125, 084102 (2006).

${ }^{22}$ C. Daday, C. König, O. Valsson, J. Neugebauer, and C. FilIPPI, J. Chem. Theory Comput. 9, 2355 (2013).

${ }^{23}$ N. Govind, Y. A. Wang, A. J. R. DA Silva, and E. A. Carter, Chem. Phys. Lett. 295, 129 (1998).

${ }^{24}$ A. S. P. Gomes, C. R. JACOB, and L. Visscher, Phys. Chem. Chem. Phys. 10, 5353 (2008).

${ }^{25}$ D. K. Kanan, S. Sharifzadeh, and E. A. Carter, Chem. Phys. Lett. 519-520, 18 (2012).

${ }^{26}$ C. Daday, C. König, J. Neugebauer, and C. Filippi, ChemPhysChem, DOI: 10.1002/cphc.201402459 (2014).

${ }^{27}$ C. R. Jacob and J. Neugebauer, WIREs Comput. Mol. Sci. 4, 325 (2014).

${ }^{28}$ F. Libisch, C. Huang, and E. A. Carter, Acc. Chem. Res., DOI: $10.1021 / \operatorname{ar} 500086 \mathrm{~h}(2014)$.

${ }^{29}$ A. S. P. Gomes and C. R. JАCOB, Annu. Rep. Prog. Chem., Sect. C: Phys. Chem. 108, 222 (2012).

${ }^{30}$ S. R. White, Phys. Rev. Lett. 69, 2863 (1992).

${ }^{31}$ S. R. White, Phys. Rev. B 48, 10345 (1993).

32 Ö. Legeza, R. M. Noack, J. Sólyom, and L. Tincani, Lect. Notes Phys. 739, 653 (2008).

${ }^{33}$ K. H. Marti and M. Reiner, Z. Phys. Chem. 224, 583 (2010).

${ }^{34}$ S. Sharma and G. K.-L. Chan, J. Chem. Phys. 136, 144105 (2012).

${ }^{35}$ G. K.-L. Chan, WIREs: Comput. Mol. Sci. 2, 907 (2012).

${ }^{36}$ Y. Kurashige, Mol. Phys. 112, 1485 (2014).
${ }^{37} \mathrm{~S}$. WOUTERS and D. VAN NECK, The density matrix renormalization group for ab initio quantum chemistry, arXiv:1407.2040, 2014.

${ }^{38}$ S. Wouters, W. Poelmans, P. W. Ayers, and D. V. Neck, Comp. Phys. Commun. 185, 1501 (2014).

${ }^{39}$ S. Knecht, O. Legeza, and M. Reiner, J. Chem. Phys. 140, 041101 (2014).

${ }^{40} \mathrm{~K}$. Boguslawski, K. H. Marti, Ö. Legeza, and M. Reiher, J. Chem. Theory Comput. 8, 1970 (2012).

${ }^{41}$ K. Boguslawski, P. Tecmer, O. Legeza, and M. Reiher, J. Phys. Chem. Lett. 3, 3129 (2012).

${ }^{42}$ K. Boguslawski, P. Tecmer, G. Barcza, Ö. Legeza, and M. Reiner, J. Chem. Theory Comput. 9, 2959 (2013).

${ }^{43}$ Y. Kurashige, G. K.-L. Chan, and T. Yanai, Nat. Chem. 5, 660 (2013).

${ }^{44}$ T. V. Harris, Y. Kurashige, T. Yanai, and K. Morokuma, J. Chem. Phys. 140, 054303 (2014).

${ }^{45}$ T. N. Lan, Y. Kurashige, and T. Yanai, J. Chem. Theory Comp. 10, 1953 (2014).

${ }^{46}$ S. Sharma, T. Yanai, G. H. Booth, C. J. Umrigar, and G. K.L. Chan, J. Chem. Phys. 140, 104112 (2014).

${ }^{47} \mathrm{P}$. Tecmer, K. Boguslawski, Ö. Legeza, and M. Reiher, Phys. Chem. Chem. Phys. 16, 719 (2014).

${ }^{48} \mathrm{~S}$. Wouters, T. Bogaerts, P. Van Der Voort, V. Van Speybroeck, and D. Van Neck, J. Chem. Phys. 140, 241103 (2014).

${ }^{49}$ K. H. Marti, I. M. Ondik, G. Moritz, and M. Reiher, J. Chem. Phys. 128, 014104 (2008).

${ }^{50}$ S. Keller and M. Reiher, Chimia 68, 200 (2014).

${ }^{51}$ B. O. Roos, in Radiation Induced Molecular Phenomena in Nucleic Acids, edited by M. K. Shukla and J. Leszczynski, pp. 125-156, Berlin, 2008, Springer

${ }^{52}$ T. A. Wesolowski, H. Chermette, and J. Weber, J. Chem. Phys. 105, 9182 (1996).

${ }^{53}$ N. Govind, Y. A. Wang, and E. A. Carter, J. Chem. Phys. 110, 7677 (1999).

${ }^{54}$ F. Aquilante, L. D. Vico, N. Ferré, G. Ghigo, P.-Å. Malmqvist, P. Neogrády, T. B. Pedersen, M. Pitonak, M. Reiher, B. Roos, L. Serrano-Andrés, M. Urban, V. Veryazov, and R. Lindh, J. Comp. Chem. 31, 224 (2010).

${ }^{55}$ S. Keller, M. Dolfi, M. Reiher, and M. Troyer, QCMAQUIS: A massively parallel, Matrix-Product-Operator based Quantum-Chemical DMRG program, in preparation, 2014.

${ }^{56} \mathrm{G}$. te Velde, F. M. Bickelhaupt, E. J. Baerends, S. J. A. van Gisbergen, C. Fonseca Guerra, J. G. Snijders, and T. Ziegler, J. Comput. Chem. 22, 931 (2001).

${ }^{57}$ J. Neugebauer, C. R. Jacob, T. A. Wesolowski, and E. J. Baerends, J. Phys. Chem. A 109, 7805 (2005).

${ }^{58}$ C. R. Jacob, J. Neugebauer, and L. Visscher, J. Comput. Chem. 29, 1011 (2008).

${ }^{59}$ M. Dolfi, B. Bauer, S. Keller, A. Kosenkov, T. Ewart, A. Kantian, T. Giamarchi, and M. Troyer, Matrix Product State applications for the ALPS project, arXiv:1407.0872, 2014.

${ }^{60}$ C. R. Jacob, S. M. Beyhan, R. E. Bulo, A. S. P. Gomes, A. W. Götz, K. Kiewisch, J. Sikkema, and L. Visscher, J. Comput. Chem. 32, 2328 (2011).

${ }^{61}$ J. P. Perdew, J. A. Chevary, S. H. Vosko, K. A. Jackson, M. R. Pederson, D. J. Singh, and C. Fiolhais, Phys. Rev. B 46, 6671 (1992).

${ }^{62}$ E. Van Lenthe and E. J. Baerends, J. Comput. Chem. 24, 1142 (2003).

${ }^{63}$ A. Lembarki and H. Chermette, Phys. Rev. A 50, 5328 (1994).

${ }^{64}$ T. H. Dunning JR., J. Chem. Phys. 90, 1007 (1989).

${ }^{65}$ F. NeEse, WIREs Comput. Mol. Sci. 2, 73 (2012).

${ }^{66}$ A. D. Becke, Phys. Rev. A 38, 3098 (1988).

67 J. P. Perdew, Phys. Rev. B 33, 8822 (1986).

${ }^{68}$ S. Grimme, J. Antony, S. Ehrlich, and H. Krieg, J. Chem. Phys. 132, (2010).

${ }^{69}$ F. Weigend and R. Ahlrichs, Phys. Chem. Chem. Phys. 7, 3297 (2005). 